\title{
Erratum to: Development of the Tetron Model
}

\author{
Bodo Lampe
}

Received: 13 January 2010 / Accepted: 14 January 2010 / Published online: 29 January 2010

(C) Springer Science+Business Media, LLC 2010

\section{Erratum to: Found Phys \\ doi:10.1007/s10701-009-9278-9}

In contrast to what was written in Sect. 6 of the paper the tetron spin problem cannot be solved by the use of octonions. Therefore another approach is briefly described here which solves the problem and leads phenomenologically to the same consequences as the original model.

The solution I suggest relies on the fact that $S_{4}$ is not only the symmetry group of a regular tetrahedron, but also of a tetrahedral lattice. It is therefore tempting to assume, that the inner symmetry space of tetrons is not continuous (with a continuous symmetry group) but has instead the discrete structure of such a tetrahedral lattice. (Models of that type have been discussed e.g. in Refs. [1, 2].) The observed quarks and leptons are then interpreted as excitations on this lattice and characterized by representations of the lattice symmetry group $S_{4}$, i.e. by $A_{1}+A_{2}+2 E+3 T_{1}+3 T_{2}$, just as before. In this picture the original dynamics is governed by some unknown lattice interaction instead of by four real tetron constituents.

The lattice ansatz also naturally explains the selection rule discussed in Sect. 4 of the paper that all physical states must be permutation states: just because only representations of the permutation group $S_{4}$ are allowed.

One has then in principle two choices. Either to leave physical space continuous or to assume that at very small distances it is a lattice, too. The latter option has been analyzed in detail in Ref. [3]. Its cosmological and astrophysical consequences have been discussed there as well.

The online version of the original article can be found under doi:10.1007/s10701-009-9278-9.

B. Lampe $(\varangle)$

Hans-Maurer-Str. 9, 84435 Lengdorf, Germany

e-mail: Lampe.Bodo@web.de 
Using an inner symmetry lattice, one gets rid of the problem that one has to make a spin- $\frac{1}{2}$ particle from 4 identical constituents. For example, one can look for 3 -fold 'elementary' excitations on the inner symmetry lattice, from which the observed spectrum can be built. Using excitations $g_{1 \text { in }}, t_{1 \text { in }}$ and $h_{\text {in }}$ (transforming with respect to the representations $G_{1}, T_{1}$ and $H$ of $S_{4}$, respectively) the quark and lepton spectrum can be built as

$$
g_{1 \text { in }} \otimes t_{1 \text { in }} \otimes h_{\text {in }}=A_{1}+A_{2}+2 E+3 T_{1}+3 T_{2}
$$

\section{References}

1. Seiler, E., Patrascioiu, A.: Phys. Rev. D 64, 065006 (2001)

2. Hasenfratz, P., Niedermayer, F.: Nucl. Phys. B 596, 481 (2001)

3. Lampe, B.: Cent. Eur. J. Phys. (2010). doi:10.2478/s11534-010-0002-3 